\title{
The Role of Artificial Intelligence (AI) in Condition Monitoring and Diagnostic Engineering Management (COMADEM): A Literature Survey
}

\author{
B. K. Nagaraja Rao \\ Comadem International, 307 Tiverton Road, Selly Oak, Birmingham, UK
}

Email address:

rajbknrao@btinternet.com

To cite this article:

B. K. Nagaraja. Rao. The Role of Artificial Intelligence (AI) in Condition Monitoring and Diagnostic Engineering Management (COMADEM): A Literature Survey. American Journal of Artificial Intelligence. Vol. 5, No. 1, 2021, pp. 17-37.

doi: 10.11648/j.ajai.20210501.12

Received: March 27, 2021; Accepted: April 9, 2021; Published: April 30, 2021

\begin{abstract}
Artificial Intelligence (AI) is playing a dominant role in the $21^{\text {st }}$ century. Organizations have more data than ever, so it's crucial to ensure that the analytics team should differentiate between Interesting Data and Useful Data. Amongst the important aspects in Machine Learning are "Feature Selection" and "Feature Extraction". We are now witnessing the emerging fourth industrial revolution and a considerable number of evolutionary changes in machine learning methodologies to achieve operational excellence in operating and maintaining the industrial assets efficiently, reliably, safely and cost-effectively. AI techniques such as, knowledge based systems, expert systems, artificial neural networks, genetic algorithms, fuzzy logic, casebased reasoning and any combination of these techniques (hybrid systems), machine learning, biomimicry such as swarm intelligence and distributed intelligence. are widely used by multi-disciplinarians to solve a whole range of hitherto intractable problems associated with the proactive maintenance management of industrial assets. In this paper, an attempt is made to review the role of artificial intelligence in condition monitoring and diagnostic engineering management of modern engineering assets. The paper also highlights that unethical and immoral misuse of $\mathrm{AI}$ is dangerous.
\end{abstract}

Keywords: Artificial Intelligence (AI), Failure Modes, Feature Selection, Feature Extraction, Condition Monitoring, Smart Decision-making, Diagnosis and Prognosis, Potential Ethical and Moral Issues

\section{Introduction}

In the first half of the 20th century, science fiction familiarized the world with the concept of artificially intelligent robots. In the $1940 \mathrm{~s}$ and $50 \mathrm{~s}$, a handful of scientists from a variety of fields (mathematics, psychology, engineering, economics and political science) began to discuss the possibility of creating an artificial brain. The field of artificial intelligence research was founded as an academic discipline in 1956. Alan Turing explored the mathematical possibility of artificial intelligence. In his 1950 paper on Computing Machinery and Intelligence he discussed how to build intelligent machines and how to test their intelligence. Lack of funding and a proof of concept were needed to persuade that machine intelligence was worth pursuing. The first artificial intelligence program was presented at the Dartmouth Summer Research Project on Artificial
Intelligence (DSRPAI) hosted by John McCarthy and Marvin Minsky in 1956. The significance of this event cannot be undermined as it catalyzed the next twenty years of AI research. From 1957 to 1974, AI flourished. Computers could store more information and became faster, cheaper, and more accessible. In the 1980's, John Hopfield and David Rumelhart popularized "deep learning" techniques which allowed computers to learn using experience. In the 1980s a form of AI program called "expert systems" was adopted by corporations around the world and knowledge became the focus of mainstream AI research. In those same years, the Japanese government aggressively funded AI with its fifth generation computer project. Another encouraging event in the early 1980s was the revival of connectionism in the work of John Hopfield and David Rumelhart. Once again, AI had achieved success. In 1997, reigning world chess champion and grand master Gary Kasparov was defeated by IBM's 
Deep Blue, a chess playing computer program. In the same year, speech recognition software, developed by Dragon Systems, was implemented on Windows. Now artificial intelligence is everywhere. We now live in the age of "big data". Today's digital transformation, also termed "the 4th industrial revolution" is data-driven. It is not about the transition from paper-written reports to pdfs, but the ability to capture and utilize vast amounts of data generated in a business context. Tomorrow's winners are said to be those that the most valuable data and know-how to capitalize on it. This area is exploding with advancements in sensor technology and IoT connectivity, enabling data generation and data capture from any device. Any digital interaction and transaction, from website interactions machinery sensor measurement, can be captured and monetized if it is placed in the right business context, solving some real business problems. Identifying, exploring, and solving such problems with data have resulted in the birth of discipline of Data Science. Artificial intelligence is now widely used in many applications such as Internet-of-things, cyber-physical systems, intelligent transportation systems, smart vehicles, big data analytics, understanding complex networks, neural networks, fuzzy systems, neuro-fuzzy systems, deep learning, real world applications, self-organizing, emerging or bioinspired systems, global optimization, meta-heuristics and their applications, evolutionary algorithms, swarm intelligence, nature and biologically inspired meta-heuristics, etc.

\section{COMADEM - A Brief History}

The philosophy of Condition Monitoring and Diagnostic Engineering Management (COMADEM) is based on "Prevention is better than Cure" and "Any quick-fix solutions are dangerous". There are two kinds of assets; (a) physical assets and (b) human assets. All assets are subjected to lifecyclic processes involving creation, sustenance and destruction. The moment an asset is created, seeds of nanofailures are automatically embedded which, over a period of time will be triggered by various means during the design, manufacturing, storage, maintenance, operational and disposal stages. Failures can be classified under the following headings: mechanical failures, structural failures, electrical/electronic failures, electro-mechanical failures, radiation related failures, human factors related failures, tribological failures, hydraulic/pneumatic failures, metallurgical failures, chemical failures, corrosion failures, meteorological failures, maintenance related failures. By proactively monitoring the performance of the assets throughout its useful life-cycle, it is possible to effectively diagnose the root cause failure modes and prevent the costly major system downtime to absolute minimum.

Back in 1980 s very little attention was given to the proactive maintenance management of industrial assets. Many industries, academic institutions and professional bodies widely believed and practiced the reactive philosophy "Fix it when it breaks down". Improper maintenance was costing the industries billions of pounds each year. It was during this time, the idea of discovering, generating and disseminating the interdisciplinary-based knowledge of proactive maintenance management was conceived. This was the birth of the multi-disciplinary and proactive philosophy of Condition Monitoring And Diagnostic Engineering Management (COMADEM). Since 1988, the philosophy of COMADEM is disseminated by hosting annual international congresses and exhibitions in many parts of the world. The 33rd International Congress and Exhibition on COMADEM will be hosted by the Lulea University of Technology in Sweden in October 2021.

Proactively diagnosing, prognosing and managing the root cause failure modes of modern industrial assets remain the greatest challenge. COMADEM provide a common platform where industrial and academic researchers from many disciplines actively participate in a friendly environment to contribute and share the latest diagnostic and prognostic knowledge and wisdom to reduce systems failure to absolute minimum with long-term maximum safety, cost benefit and reliability. By implementing this proactive philosophy, many companies have reaped benefits by minimizing the downtime, cost of repairs, and optimizing design of future systems. COMADEM is now widely recognized by many world-class universities, research and development organizations and industries as a cost-effective proactive management multi discipline in its own right.

\section{Artificial Intelligence Techniques Employed in COMADEM}

Smart systems and artificial intelligence are playing an ever-increasing role in maintaining our industrial engineering assets. Modern engineering systems are highly sophisticated and complex in nature. Operating and maintaining these systems reliably, safely and cost-effectively poses a number of challenging problems. Proactively monitoring various critical performance parameters, diagnosing/prognosing the root cause failures involves up to date knowledge and decision making skills. AI is not one universal technology, it is rather an umbrella term that includes multiple technologies such as machine learning, compressive sampling, learning algorithms, classification algorithms, reasoning, IoT, Industry 4.0, computer vision and natural language processing (NLP) to allow for a more intricate and comprehensive approach to the design, production and optimization of assembly and production components. The broadness of the technology portfolio, its rapid pace of change, and the increasing power of the proposed solutions leverages computer-aided design and manufacturing, sensing systems, data and physics based models, fault prediction and diagnosis, and data visualization to create a virtual asset management toolbox. Artificial Intelligence technologies have the potential to assist engineering industry to achieve disruptive transformations both in performance and in value generation. Artificial Intelligence is used to describe machines that mimic 
"cognitive" functions that humans associate with other human minds, such as "learning" and "problem solving". With better availability of information, operators and plant controllers can make improved decisions, accelerate human/operators learning, enable the human workforce to be more effective and efficient as well as more creative, reduce human involvement in physical tasks in dangerous working environments, improve the operators' working conditions by simplification of human-machine interface with complex processes and transfer and formalize operators' knowledge and best practices.

In the following some of the AI techniques employed in the proactive discipline of COMADEM is briefly discussed.

(a) Knowledge-based Systems: A knowledge-based system (KBS) is a form of artificial intelligence (AI) that aims to capture the knowledge of human experts to support decision-making. Examples of knowledge-based systems include expert systems, which are so called because of their reliance on human expertise. The typical architecture of a knowledge-based system, which informs its problem-solving method, includes a knowledge base and an inference engine. The knowledge base contains a collection of information in a given field medical diagnosis, for example. The inference engine deduces insights from the information housed in the knowledge base. Knowledge-based systems also include an interface through which users query the system and interact with it. Over the years, knowledge-based systems have been developed for a number of applications including industrial equipment fault diagnosis. Daniel Dennett, a philosopher and cognitive scientist, in his 2017 book, From Bacteria to Bach and Back, cited a strategy shift from early AI, characterized by "top-down-organized, bureaucratically efficient know-it-all" systems to systems that harness Big Data and "statistical pattern-finding techniques" such as data-mining and deep learning in a more bottom-up approach. Examples of AI following the latter approach include neural network systems, a type of deep-learning technology that concentrates on signal processing and pattern recognition problems such as facial recognition. The topics that are covered include (a) Big data techniques and methodologies, data-driven information systems, and knowledge acquisition, (b) Cognitive interaction and intelligent human interfaces, (c) Recommender systems and E-service personalization, (d) Intelligent decision support systems, prediction systems and warning systems, (e) Computational and artificial intelligence based systems and uncertain information processes, (f) Swarm intelligence and evolutionary computing, (g) Knowledge engineering, machine learning-based systems and web semantics. Fault classification and evaluation is a complex logical process that requires intelligent decision making techniques. Therefore, knowledge based methods are of great importance in fault diagnosis and expert systems have so far been applied successfully for fault diagnosis (Frank [29]). A number of KBS were used for maintenance and fault diagnosis. A survey on the use of KBS for failure diagnosis before 1993 can be found in (Rowland [76]). Arslan et al.. [1] reported the use of an expert system for failure diagnosis for printed circuit boards. The KBS exploits functional test data, which is output from automatic test equipment which is used to test every board subsequent to manufacture. Fujikawa and Ishii [30] use a KBS to identify the causes of various manufacturing defects in hot forging and suggest remedies. For maintenance, several systems have been developed to tackle specific industrial applications. For example Clark et al [17] developed a knowledge-based system to optimize the building management maintenance and Batanov et al [7] developed EXPERTMM: a KBS for maintenance management for a large manufacturing company. A more general approach based on knowledge-based reasoning was adopted by Kobacy et al [50] and Zhang and Jardine(1997). Kobacy et al [50] developed IMOS, a prototype intelligent maintenance optimization system aimed at developing and enhancement of preventive maintenance routines for large and complex industrial systems. IMOS has a rule base for selecting an appropriate model for application based on identification of maintenance data pattern. Zhang and Jardine [99] proposed a similar smart system for dataanalysis models and optimizing replacement. ShuHsien Liao [78] surveys and classifies KM technologies using the seven categories as: KM framework, knowledge-based systems, data mining, information and communication technology, artificial intelligence/expert systems, database technology, and modelling, together with their applications for different research and problem domains. Some discussion is presented, indicating future development for knowledge management technologies and applications as the followings: (1) KM technologies tend to develop towards expert orientation, and KM applications development is a problem-oriented domain. (2) Different social studies methodologies, such as statistical method, are suggested to implement in KM as another kind of technology. (3) Integration of qualitative and quantitative methods, and integration of KM technologies studies may broaden our horizon on this subject. (4) The ability to continually change and obtain new understanding is the power of $\mathrm{KM}$ technologies and will be the application of future works. Rene Peinl [75] highlights those disruptions by discussing three recent trends in the IT sector (namely DevOps, Internet of Things and deep learning, which all have dedicated tracks at e.g. Cloud Expo 2017) and explaining the implications when transferring the insights from IT to Knowledge Workers (KWs) in general. The goal is to create a better understanding of the future of knowledge management by using 
inductive reasoning to infer recommended actions for KWs in general from insights from IT industry. Yang $\mathrm{Lu}$ [98] conducts a comprehensive review on Industry 4.0 and presents an overview of the content, scope, and findings of Industry 4.0 by examining the existing literatures in all of the databases within the Web of Science. Altogether, 88 papers related to Industry 4.0 are grouped into five research categories and reviewed. In addition, this paper outlines the critical issue of the interoperability of Industry 4.0, and proposes a conceptual framework of interoperability regarding Industry 4.0. Challenges and trends for future research on Industry 4.0 are discussed.

(b) Artificial Neural Networks: Artificial Neural networks (ANNs) are a set of algorithms, modelled loosely after the human brain, that are designed to recognize patterns. They interpret sensory data through a kind of machine perception, labelling or clustering raw input. Neural networks help to cluster and classify. The three major learning paradigms are supervised learning, unsupervised learning and reinforcement learning. All classification tasks depend upon labelled datasets; that is, humans must transfer their knowledge to the dataset in order for a neural network to learn the correlation between labels and data. This is known as supervised learning. Clustering or grouping is the detection of similarities. Deep learning does not require labels to detect similarities. Learning without labels is called unsupervised learning. With classification, deep learning is able to establish correlations between, say, pixels in an image and the name of a person. This might be called as a static prediction. By the same token, exposed to enough of the right data, deep learning is able to establish correlations between present events and future events. It can run regression between the past and the future. The future event is like the label in a sense. Deep learning doesn't necessarily care about time, or the fact that something hasn't happened yet. We're also moving toward a world of smarter agents that combine neural networks with other algorithms like reinforcement learning to attain goals. Deep-learning networks perform automatic feature extraction without human intervention, unlike most traditional machine-learning algorithms. In some circles, neural networks are thought of as "brute force" AI, because they start with a blank slate and hammer their way through to an accurate model. NNs have been widely used in monitoring and diagnosing (Zhang and Huang, [100]. NNs have been suggested as alternatives to state process charts, and shown to exhibit superior performance in detecting non-random patterns (Chang and Ho [16]. Researchers are working to combine monitoring of process means with monitoring of process variance (e.g. Chang and Ho [16], Ho and Chang [40], Dedeakayogullari and Buma, [18]. The ability of NNs to detect several patterns occurring simultaneously is also being investigated (Guh and
Tannock, [31]. NNs have been used for matching stereoscopic pictures and correcting 3D measurement error (Tien and Chang [87]) extending earlier work by $\mathrm{Su}$ et al. [79] and identifying product defects (e.g. Kim and Kumara [49]; Wang and Huang [94]). A feed forward $\mathrm{NN}$ has been used for manufacturing diagnosis by Ransing and Lewis [74]. Maurizio Bevilacqua et al [66] propose a multi-layer perception based artificial neural network (MLP - ANN) as a practical instrument to evaluate the expected failure rates of 143 centrifugal pumps used in an oil refinery plant. Design/methodology/approach - A MLP is adopted to weigh up the correlation existing among the failure rates and the several different operating conditions which have some influence in the occurrence. The findings reveal that during the training phase, it is possible to discriminate among those variables closely significant for the final outcome and those which can be kept off from the analysis. In particular, the neural network automatically calculates and classifies the centrifugal pumps in terms of both the failure probability and its variability degree, giving a better analysis instrument to take decisions and to justify them, in order to optimize and fully support an eventual preventive maintenance (PM) program. The authors claim that their approach aids in decision making to reduce the necessity of reactive maintenance activities and to simplify the planning of PM ones. Mateusz Dybkowski1 and Kamil Klimkowski [61] describes a Fault Tolerant Control structure for the Induction Motor (IM) drive. They analyzed the influence of current sensor faults on the properties of the vector-controlled IM drive system. As a control algorithm, the Direct Field Oriented Control structure was chosen. For the proper operation of this system and for other vector algorithms, information about the stator currents components is required. It is important to monitor and detect these sensor faults, especially in drives with an increased safety level. They discuss the possibility of the neural network application in detecting stator current sensor faults in the vector control algorithm. Simulation and experimental results for various drive conditions are presented. Zhe Li et al [101] investigated fault diagnosis and prognosis in machine centers based on data mining approaches to formulate a systematic approach and obtain knowledge for predictive maintenance in Industry 4.0 era. They introduced a system framework based on Industry 4.0 concepts, which includes the process of fault analysis and treatment for predictive maintenance in machine centers. The framework includes five modules: sensor selection and data acquisition module, data preprocessing module, data mining module, decision support module, and maintenance implementation module. Furthermore, a case study was presented to illustrate the application of the data mining methods for fault diagnosis and prognosis in machine centers as an 
Industry 4.0 scenario. Hans R. DePold and F. Douglas Gass [39] presented recent developments in technology and strategies in engine condition monitoring including: 1) application of statistical analysis and artificial neural network filters to improve data quality; 2) neural networks for trend change detection, and classification to diagnose performance change; and 3) expert systems to diagnose, provide alerts and to rank maintenance action recommendations.

(c) Fuzzy Logic: Fuzzy logic (FL) is an approach to computing based on "degrees of truth" rather than the usual "true or false" (1 or 0 ) Boolean logic on which the modern computer is based. It is employed to handle the concept of partial truth, where the truth value may range between completely true and completely false. Fuzzy logic has been used in numerous applications such as facial pattern recognition, air conditioners, washing machines, vacuum cleaners, antiskid braking systems, transmission systems, control of subway systems and unmanned helicopters, knowledge-based systems for multi objective optimization of power systems, etc. Fuzzy set theory has been used in commercial applications of expert systems and control devices for trains and elevators; it has also been combined with neural nets to control the manufacture of semiconductors. By incorporating fuzzy logic and fuzzy sets in production systems, significant improvements have been gained in many AI systems. This approach has been particularly successful with ambiguous data sets or when the rules are imperfectly known. Despite its commercial success, fuzzy logic remains a controversial idea within the artificialintelligence community. Many researchers question the consistency and validity of the methods used to 'reason' with fuzzy logic. Fault identification can be seen as a classification problem. The task is to match each pattern of the symptom vector with one of the preassigned classes of faults. The principle of fault evaluation using fuzzy logic consists of a three-step process. First the symptoms have to be fuzzified, then they have to be evaluated by an inference mechanism using fuzzy IF-THEN rules, and finally they have to be defuzzified (Frank [29]). The fuzzification of the signals is a mapping of the representation using crisp values into a representation by fuzzy sets. With the aid of a fuzzy relation, the set of the fuzzified signals is transformed into a set of fuzzy statements (faults). Finally, the fuzzy information on the faults has to be converted into crisp sets such as yes-no statements. Khoo et al. [48] propose the use of fuzzy logic techniques rather than $\mathrm{NNs}$, which require long training times and large amounts of data, or KBS, which are domain dependent and have difficulty dealing with novel situations. A. Soliman et al [80] presented a scheme and methodology for integrating subjective (heuristic) and objective (analytical) knowledge for fault diagnosis and decision-making using fuzzy logic. The structure, challenges, and benefits of such integration are explored. Also, experimental results of the work carried out are presented. Timothy J. Ross [77] summarizes only two popular methods of classification. The first is classification using equivalence relations. This approach makes use of certain special properties of equivalence relations and the concept of defuzzification known as lambda-cuts on the relations. The second method of classification is a very popular method known as fuzzy c-means (FCM), so named because of its close analogue in the crisp world, hard c-means (HCM). This method uses concepts in n-dimensional Euclidean space to determine the geometric closeness of data points by assigning them to various clusters or classes and then determining the distance between the clusters. In the case of fuzzy relations, for all fuzzy equivalence relations, their ?-cuts are equivalent ordinary relations. Hence, to classify data points in the universe using fuzzy relations, we need to find the associated fuzzy equivalence relation. fuzzy logic; pattern clustering. Haitham Ramadan [38] has developed fault Diagnosis model and Remnant Life Estimation model using diagnostics and data interpretation techniques based on fuzzy logic technique, These models take a set of tests results of dissolved gas analysis, 2furaldehyde (2FAL) concentration, 2-furaldhyde(2FAL rate), water content, acidity, temperature rise, and interfacial tension (IFT), etc., This model allow better planning, maintenance approaches well as predicting the remnant life of the asset within a practical accuracy. A reliable machine fault prognostic system can be used to forecast damage propagation trend in rotary machinery and to provide an alarm before a fault reaches critical levels. Currently, there are several techniques available in the literature for time-series prediction. Among the most promising methods are recurrent neural networks (RNNs) and neuro-fuzzy (NF) systems. The performance of these two types of predictors is evaluated by Wilson Q. Wang, M. Farid, Golnaraghi and Fathy Ismail [95] using two benchmark data sets. Through comparison it is found that if an NF system is properly trained, it performs better than RNNs in both forecasting accuracy and training efficiency. Accordingly, NF system is adopted to develop an on-line machine fault prognostic system. In order to facilitate the automatic monitoring process, reference function approach is proposed here to enhance feature representation. The performance of the developed prognostic system is evaluated by using three test cases including a worn gear, a chipped gear, and a cracked gear, as well as using data sets from previous studies corresponding to a gear pitting damage and a shaft misalignment. From these tests, the NF prognostic system is found to be a very reliable and robust machine health condition predictor. It can capture the system dynamic behavior quickly and 
accurately. Rafik Mahdaoui and Leila Hayet Mouss [73] presented some basic aspects of TSK (Takigi Sugeno Kang) neuro-fuzzy techniques for the prognosis and diagnosis of manufacturing systems. In particular, a neuro-fuzzy model that can be used for the identification and the simulation of faults prognosis models is described. The presented model is motivated by a cooperative neuro-fuzzy approach based on a vectorized recurrent neural net-work architecture. The neuro-fuzzy architecture maps the residuals into two classes: a one of fixed direction residuals and another one of faults belonging to rotary kiln. Ivica Petrović et al [42] presented an overview of possibilities for using fuzzy logic in an assessment of primary faults in the transmission grid. The tool for this task is SCADA system, which is based on information of currents, voltages, events of protection devices and status of circuit breakers in the grid. The function model described with the membership function and fuzzy logic systems is presented in this paper. For input data, diagnostics system uses information of protection devices tripping, states of circuit breakers and measurements of currents and voltages before and after faults. M Muharam and M Latif [59] reported the early detection of machine failure to improve the performance of the production process. The Poka-Yoke device was developed to monitor the machine. The vibration signal is captured by sensors and inputted in Poka-yoke device for processing. Poka-Yoke device has two components, Fuzzy-Neural Network identification and decision maker. The first component, the time-domain signal is transformed into the frequency domain, magnitude and frequency are treated as Fuzzy membership functions by using the statistical parameters as mechanical harmonic distortion and then are trained by Neural Network. The second component, the decision is in the form of machine condition statements such as normal, alarm, and shutdown. Simulation's results show that the method can be applied to identify the machine condition in term of bearing faults. Moreover, the Poka-yoke system that developed can be used to monitor machine condition automatically.

(d) Genetic Algorithms: Genetic algorithms (GAs) were developed in the mid-1960s. Genetic algorithms are a method of optimization involving iterative search procedures based on an analogy with the process of natural selection (Darwinism) and evolutionary genetics. Genetic algorithms (GA) like neural networks are biologically inspired and represent a new computational model having its roots in evolutionary sciences. Usually GAs represent an optimization procedure in a binary search space, and unlike traditional hill climbers they do not evaluate and improve a single solution but a set of solutions or hypotheses, a so-called population. The GAs produce successor hypotheses by mutation and recombination of the best currently known hypotheses. Thus, at each iteration a part of the current population is replaced by offspring of the most fit hypotheses. In other words, a space of candidate hypotheses is searched in order to identify the best hypothesis, which is defined as the optimization of a given numerical measure, the socalled hypothesis fitness. There are several different algorithms for the solution of multi objective optimization problems and these group mostly into three basic approaches: (a) The aggregation method, which transfers the multi objective function into a single objective, (b) The criteria method, which optimizes one objective at a time and (c) The Pareto method, which uses the Pareto optimal idea to find an optimal front from which the optimal solution can be selected. The genetic algorithm uses three main types of rules at each step to create the next generation from the current population: (a) Selection rules select the individuals, called parents, that contribute to the population at the next generation, (b) Crossover rules combine two parents to form children for the next generation, (c) Mutation rules apply random changes to individual parents to form children. B. Samanta, K. R. Al-Balushi and S. A. Al-Araimi [81] compared the performance of bearing fault detection using two different classifiers, namely, artificial neural networks (ANNs) and support vector machines (SMVs). The time-domain vibration signals of a rotating machine with normal and defective bearings are processed for feature extraction. The extracted features from original and pre-processed signals are used as inputs to the classifiers for two-class (normal or fault) recognition. The classifier parameters, e.g., the number of nodes in the hidden layer in case of ANNs and the radial basis function kernel parameter (width) in case of SVMs along with the selection of input features are optimized using genetic algorithms. The classifiers are trained with a subset of the experimental data for known machine conditions and are tested using the remaining set of data. The procedure is illustrated using the experimental vibration data of a rotating machine. The roles of different vibration signals and signal preprocessing techniques are investigated. The results show the effectiveness of the features and the classifiers in detection of machine condition. Lei Lu et al [55] proposed a dominant feature selection scheme to enable the high performance prognostics of machine health. Statistical features were extracted from decomposed sub-modes by wavelet transform. Fisher ratio was employed to evaluate the extracted feature vectors, and dynamic searching strategy-based genetic algorithm was used to select the optimal feature subsets on the basis of maximizing the fitness function. Then dominant features with minimum mean square errors were used to predict the performance of machine health. Experimental results on predicting the lifetime of an unbalance vibration rotor system demonstrated that the 
proposed method can achieve better prognosis performance with less predicting errors. Jianbin Xiong et al [43] propose a parameter optimization algorithm for the SVM based on multi-genetic algorithm. The algorithm optimizes the correlation kernel parameters of the SVM using evolutionary search principles of multiple swarm genetic algorithms to obtain a superior SVM prediction model. The experimental results demonstrate that by combining the genetic algorithm and SVM algorithm, fault diagnosis can be effectively realized for bearings of rotating machinery. Erfan Ahadi et al [26] review the applications of some popular AI methods such as artificial neural network (ANN), support vector machine (SVR) and genetic algorithm (GA) on prognostics of rotating machineries is reviewed. Literature is analyzed based on classification of these methods in order to highlight their main concepts and applications. The findings show the advantages, disadvantages, limitations and research gaps as well as future direction of research. Matteo D. L. Dalla Vedova, et al [58] propose a modelbased fault detection and isolation (FDI) method, employing a Genetic Algorithm (GA) to identify failure precursors before the performance of the system starts being compromised. Four different failure modes are considered: dry friction, backlash, partial coil short circuit, and controller gain drift. The method presented in this work is able to deal with the challenge leveraging the system design knowledge in a more effective way than data-driven strategies, and requires less experimental data. To test the proposed tool, a simulated test rig was developed. Two numerical models of the EMA were implemented with different level of detail: A high fidelity model provided the data of the faulty actuator to be analyzed, while a simpler one, computationally lighter but accurate enough to simulate the considered fault modes, was executed iteratively by the GA. The results showed good robustness and precision, allowing the early identification of a system malfunctioning with few false positives or missed failures. $\mathrm{N}$ Belu et al [6] presents a risk-cost analysis model that uses Genetic algorithms to generate an FMEA for the raw materials and finished parts reception process, in automotive industry. Unlike the classic FMEA analysis, in their model the risk factors $\mathrm{D}$ and $\mathrm{O}$ are determined by resources cost analysis involved in their improvement. Here comes the Genetic algorithm that will determine the optimal cost under an acceptable risk. The proposed solution uses modern information technology for data acquisition (complex event processing), automation of analysis process (artificial intelligence) and long-term support for quality staff in FMEA analysis (real time and batch data analytics).

(e) Case Based Reasoning: Case-based reasoning (CBR), broadly construed, is the process of solving new problems based on the solutions of similar past problems. Case-based reasoning is based on the paradigm of human thought in cognitive psychology that contends that human experts derive their knowledge from solving numerous cases in their problem domain. Case-based reasoning has been formalized for purposes of computer reasoning as a four-step process: (a) Retrieve: Given a target problem, retrieve from memory cases relevant to solving it. A case consists of a problem, its solution, and, typically, annotations about how the solution was derived, (b) Reuse: Map the solution from the previous case to the target problem. This may involve adapting the solution as needed to fit the new situation. (c) Revise: Having mapped the previous solution to the target situation, test the new solution in the real world (or a simulation) and, if necessary, revise. (d) Retain: After the solution has been successfully adapted to the target problem, store the resulting experience as a new case in memory. A possible application of case-based reasoning to concrete problems is the identification of causes of concrete deterioration where the records are incomplete and traditional methods of analysis are inconclusive. Xia and Rao (Xia and Rao [97]) developed a dynamic CBR system that can represent system dynamics and fault-propagation. The CBR include mechanisms such as tagged indexes, dynamic and composite features, and multiple indexing paths. Ziyan Wen et al [102] have developed a distributed diagnostic agent system, DDAS that detects faults of a device based on signal analysis and machine learning. The CBR techniques presented are used to find root cause of vehicle faults based on the information provided by the signal agents in DDAS. Two CBR methods are presented, one used directly the diagnostic output from the signal agents and another uses the signal segment features. Experiments conducted on real vehicle cases collected from auto dealers and the results show that both methods are effective in finding root causes of vehicle faults. In Discrete Event System, such as railway onboard system, overwhelming volume of textual data is recorded in the form of repair verbatim collected during the fault diagnosis process. Efficient text mining of such maintenance data plays an important role in discovering the best-practice repair knowledge from millions of repair verbatims, which help to conduct accurate fault diagnosis and predication. In order to improve the traditional mechanical fault diagnosis expert system, case-based reasoning (CBR) is introduced by Jun-Jie [44] for fault diagnosis and a CBR fault diagnosis model is established. In the process of case retrieval, an improved grey correlation model is used to calculate similarity between cases. This model can accurately retrieve similar cases and improve the speed of case retrieval. Finally, a bearing fault diagnosis expert system is established with the method proposed in this paper. Zhiwang Zhong et al [103] presents a text case-based reasoning framework 
by cloud computing, which uses the diagnosis ontology for annotating fault features recorded in the repair verbatim. The extracted fault features are further reduced by rough set theory. Finally, the case retrieval is employed to search the best-practice repair actions for fixing faulty parts. By cloud computing, rough setbased attribute reduction and case retrieval are able to scale up the Big Data records and improve the efficiency of fault diagnosis and predication. The effectiveness of the proposed method is validated through a fault diagnosis of train onboard equipment. A model to implement the case-based reasoning developed from using a neural net as a base to calculate a measure of similarity between the new problem and each case is presented by María Matilde García Lorenzo and Rafael Estebán Bello Pérez [56]. It is shown how the neural net provides a mechanism to retrieve cases considering information that in other models needs a parallel architecture. The author has analyzed how the model can be employed to create expert networks and case-based planners. Zhiwang Zhong et al [103] presents a text case-based reasoning framework by cloud computing, which uses the diagnosis ontology for annotating fault features recorded in the repair verbatim. The extracted fault features are further reduced by rough set theory. Finally, the case retrieval is employed to search the bestpractice repair actions for fixing faulty parts. By cloud computing, rough set-based attribute reduction and case retrieval are able to scale up the Big Data records and improve the efficiency of fault diagnosis and predication. The effectiveness of the proposed method is validated through a fault diagnosis of train onboard equipment. Cordelia Mattuvarkuzhali Ezhilarasu et al [15] discuss the importance and the necessity of reasoning applications in the field of Aerospace Integrated Vehicle Health Management (IVHM). A fully functional IVHM system is required to optimize Condition Based Maintenance (CBM), avoid unplanned maintenance activities and reduce the costs inflicted there upon. This IVHM system should be able to utilize the information from multiple subsystems of the vehicle to assess the health of those subsystems, their effect on the other subsystems, and on the vehicle as a whole. Such a system can only be realized when the supporting technologies like sensor technology, control and systems engineering, communications technology and Artificial Intelligence (AI) are equally advanced. This paper focuses on the field of AI, especially reasoning technology and explores how it has helped the growth of IVHM in the past. The paper reviews various reasoning strategies, different reasoning systems, their architectures, components and finally their numerous applications. The paper discusses the shortcomings found in the IVHM field, particularly in the area of vehicle level health monitoring and how reasoning can be applied to address some of them. It also highlights the challenges faced when the reasoning system is developed to monitor the health at the vehicle level and how a few of these challenges can be mitigated.

(f) Hybrid Systems: Hybrid systems (HS) arise when the continuous and the discrete meet. Combine continuous and discrete inputs, outputs, states, or dynamics, and we have a hybrid system. Particularly, hybrid systems arise from the use of finite-state logic to govern continuous physical processes (as in embedded control systems) or from topological and network constraints interacting with continuous control (as in networked control systems). Hybrid systems theory studies the behavior of dynamical systems, including the technological systems mentioned above described by modelling formalisms that involve both continuous models such as differential or difference equations describing the physical and mechanical part, and discrete models such as finite-state machines or Petri Nets that describe the software and logical behavior. Hybrid systems arise in embedded control when digital controllers, computers, and subsystems modelled as finite-state machines are coupled with controllers and plants modelled by partial or ordinary differential equations or difference equations. Thus, such systems arise whenever one mixes logical decision making with the generation of continuous-valued control laws. Jeon (2000) developed a hybrid intelligent maintenance optimization system (HIMOS) for decision support which aims at overcoming the problems of IMOS. The key to this analysis is the application of intelligent judgement in selecting the most suitable model from a model-base utilizing a hybrid knowledge/case based system (KBS/CBR). Thus initially a rule base is applied to select a model. If no model is matched, the system reverts to its historical case-base to match the current case with a similar case that has been previously modelled. This double reasoning adds to the system's true learning capabilities (intelligence) and increases the rate of success of model selection significantly. Rui G. Silva et al [83] developed an intelligent condition monitoring system able to detect when a cutting tool was worn out. To accomplish this objective the use of a hybrid intelligent system, based on an expert system and two neural networks was investigated. The neural networks were employed to process data from sensors and the classifications made by the neural networks were combined with information from the knowledge base to obtain an estimate of the wear state of the tool by the expert system. The novelty of this work is mainly associated with the configuration of the developed system. The combination of sensor-based information and inference rules, results in an on-line system that can learn from experience and update the knowledge base pertaining to information associated with different cutting conditions. The neural networks resolved the problem 
of interpreting the complex sensor inputs while the expert system, by keeping track of previous success, estimated which of the two neural networks was more reliable. Mis-classifications were filtered out through the use of a rough but approximate estimator, Taylor's tool life model. The system's modular structure would make it easy to update as required for different machines and/or processes. The use of Taylor's tool life model, although weak as a tool life estimator, proved to be crucial in achieving higher performance levels. The application of the Self Organizing Map to tool wear monitoring proved to be slightly more reliable then the Adaptive Resonance Theory neural network although overall the system made reliable, accurate estimates of the tool wear. Labib et al [54] used fuzzy logic combined with a rule base to develop an 'Intelligent Maintenance Model' which is applied to a manufacturing company to identify the most critical machines and determine appropriate maintenance action. Edmundas Kazimieras Zavadskas et al [25] have developed a new approach of hybrid MCDM methods and this study is limited solely on papers referred in Thomson Reuters Web of Science Core Collection academic database. It aims to review how the papers have been distributed by period of publishing and by country, which multiple criteria decision making methods have been used the most frequently in developing hybrid approaches and in what domains the methods have been applied. For a more detailed analysis of applications, journal articles from engineering research area were grouped by research domains and further by analyzed issues. Findings of the current review paper confirm that hybrid MCDM approaches, due to their abilities in integrating different techniques, can assist in handling miscellaneous information taking into account stakeholders' preferences when making decisions in engineering. In the era of big data, the ease of getting, storing and processing data is crucial. However, the trend towards big data is not as effective in the field of condition monitoring as in others. One of the challenges of today's condition monitoring is the lack of data on those assets not allowed to operate beyond their pre-established maintenance limit. Datasets miss advanced degradation states of assets and fail to predict rarely occurring outliers, but both have a great impact on operation; in other words, data-driven methods are limited and cannot accurately tackle scenarios outside the training data set. In his thesis Urko Leturiondo Zubizarreta [90] propose augmenting such datasets with the addition of synthetic data generated by physics-based models describing the behavior of assets. It argues a combination of physics-based and datadriven modelling, known as hybrid modelling, can overcome the aforementioned limitations. It proposes an architecture for hybrid modelling, based on data fusion and context awareness and oriented to diagnosis and prognosis. Urko Leturiondo et al [91] proposes an architecture to implement hybrid modelling based on the fusion of real data and synthetic data obtained in simulations using a physics-based model. This architecture has two levels of analysis: an online process carried out locally and virtual commissioning performed in the cloud. The former results in failure detection analysis to avoid upcoming failures whereas the latter leads to both diagnosis and prognosis. The proposed hybrid modelling architecture is validated in the field of rotating machinery using time-domain and frequency-domain analysis. A multi-body model and a semi-supervised learning algorithm are used to perform the hybrid modelling. The state of a rolling element bearing is analyzed and accurate results for fault detection, localization and quantification are obtained. The contextual information increases the accuracy of the results; the results obtained by the model can help improve maintenance decision making and production scheduling. Future work includes a prescriptive analysis approach. Ion Matei et al [41] present a diagnosis solution when only a partially validated model is available. The solution uses a fault-augmented physics-based model to extract meaningful behavioral features corresponding to the normal and abnormal behavior. These features together with experimental training data are used to build a data-driven statistical model used for classifying the behavior of the system based on observations. They apply this approach for a railway switch diagnosis problem.

(g) Machine Learning: The two most important aspects of machine learning (ML) are Feature Selection and Feature Extraction (Mehul Ved [57]. Machine learning is the scientific study of algorithms and statistical models that computer systems use to perform a specific task without using explicit instructions, relying on patterns and inference instead. It is seen as a subset of artificial intelligence. Machine learning is an application of artificial intelligence (AI) that provides systems the ability to automatically learn and improve from experience without being explicitly programmed. Machine learning focuses on the development of computer programs that can access data and use it learn for themselves. The process of learning begins with observations or data, such as examples, direct experience, or instruction, in order to look for patterns in data and make better decisions in the future based on the examples that we provide. The primary aim is to allow the computers learn automatically without human intervention or assistance and adjust actions accordingly. Machine learning algorithms are often categorized as supervised, semi-supervised, unsupervised or reinforced. Combining machine learning with $\mathrm{AI}$ and cognitive technologies can make it even more effective in processing large volumes of information. In a nutshell, machine learning (a) learns patterns from data, i.e. without being explicitly 
programmed, (b) makes predictions on new data, e.g. a class (classification), a numeric value (regression), or finding natural groups in data (clustering), (c) this requires 1) data, 2) a model, 3) a loss function and 4) an optimization procedure by minimizing the loss function using the data one fits the model. Some of the commonly used data science and machine learning models are; a) Principle Component Analysis (PCA), b) K-Means Clustering, c) Linear Regression, d) Logistic Regression (Classification), e) Tree Based Methods, f) Artificial Neural Networks, g) Deep Learning. Oluseun Omotola Aremu et al [65] have explored a standard of practice in regards to usage of asset data to develop machine learning (ML) analytic tools for Predictive Maintenance (PdM). They have proved through research initiatives that the data structure used to train and test ML algorithms has a great impact on their performance and accuracy. They conclude that by using poorly trained models for estimation due to improper data usage, can leave some AI-based PdM tools vulnerable to high rates of inaccurate estimations. Thus, leading to value loss during an asset's life cycle. Samir Khan and Takehisa Yairi [84] presents a systematic review of artificial intelligence based system health management with an emphasis on recent trends of deep learning within the field. Various architectures and related theories are discussed to clarify its potential. Based on the reviewed work, deep learning demonstrates plausible benefits for fault diagnosis and prognostics. However, there are a number of limitations that hinder its widespread adoption and require further development. Attention is paid to overcoming these challenges, with future opportunities being enumerated. Linxia Liao and Hyung-il Ahn [53] propose a method to integrate feature extraction and prediction as a single optimization task by stacking a three-layer model as a deep learning structure. The first layer of the deep structure is a Long Short Term Memory (LSTM) model which deals with the sequential input data from a group of assets. The output of the LSTM model is followed by mean-pooling, and the result is fed to the second layer. The second layer is a neural network layer, which further learns the feature representation. The output of the second layer is connected to a survival model as the third layer for predicting asset health condition. The parameters of the three-layer model are optimized together via stochastic gradient decent. The proposed method was tested on a small dataset collected from a fleet of mining haul trucks. The model resulted in the "individualized" failure probability representation for assessing the health condition of each individual asset, which well separates the in-service and failed trucks. The proposed method was also tested on a large open source hard drive dataset, and it showed promising result. Zhe $\mathrm{Li}$ et al [101] propose a novel deep learning-based method for anomaly detection in mechanical equipment by combining two types of deep learning architectures, stacked autoencoders (SAE) and long short-term memory (LSTM) neural networks, to identify anomaly condition in a completely unsupervised manner. The proposed method focuses on the anomaly detection through multiple features sequence when the history data is unlabeled and the empirical knowledge about anomaly is absent. An experiment for anomaly detection in rotary machinery through wavelet packet decomposition (WPD) and data-driven models demonstrates the efficiency and stability of the proposed approach. The method can be divided into two stages: SAE-based multiple features sequence representation and LSTM-based anomaly identification. During the experiment, fivefold crossvalidation has been applied to validate the performance and stability of the proposed approach. The results show that the proposed approach could detect anomaly working condition with $99 \%$ accuracy under a completely unsupervised learning environment and offer an alternative method to leverage and integrate features for anomaly detection without empirical knowledge. Maryam M Najafabadi, Flavio Villanustre, Taghi M Khoshgoftaar, Naeem Seliya, Randall Wald \& Edin Muharemagic [63] explore how Deep Learning can be utilized for addressing some important problems in Big Data Analytics, including extracting complex patterns from massive volumes of data, semantic indexing, data tagging, fast information retrieval, and simplifying discriminative tasks. They also investigated some aspects of Deep Learning research that need further exploration to incorporate specific challenges introduced by Big Data Analytics, including streaming data, high-dimensional data, scalability of models, and distributed computing. They concluded by presenting insights into relevant future works by posing some questions, including defining data sampling criteria, domain adaptation modelling, defining criteria for obtaining useful data abstractions, improving semantic indexing, semi-supervised learning, and active learning. Hosameldin Ahmed and Asoke Nandi [37] have published a book which provides an extensive, up-todate treatment of new methods including machine learning and compressive sampling, which help to improve safety, reliability and performance of rotating machines. Toyosi T. Ademujimi et al [88] focus on a review of recent fault diagnosis applications in manufacturing that are based on several prominent machine learning algorithms. Papers published from 2007 to 2017 were reviewed and keywords were used to identify 20 articles spanning the most prominent machine learning algorithms. Most articles reviewed consisted of training data obtained from sensors attached to the equipment. The training of the machine learning algorithm consisted of designed experiments to simulate different faulty and normal processing conditions. The areas of application varied from wear of cutting tool in computer numeric control (CNC) 
machine, surface roughness fault, to wafer etching process in semiconductor manufacturing. In all cases, high fault classification rates were obtained. As the interest in smart manufacturing increases, this review serves to address one of the cornerstones of emerging production systems. Shen Zhang et al systematically summarize existing literature on bearing fault diagnostics with machine learning (ML) and data mining techniques. While conventional ML methods, including artificial neural network (ANN), principal component analysis (PCA), support vector machines (SVM), etc., have been successfully applied to the detection and categorization of bearing faults for decades, recent developments in deep learning (DL) algorithms in the last five years have sparked renewed interest in both industry and academia for intelligent machine health monitoring. In this paper, we first provide a brief review of conventional ML methods, before taking a deep dive into the state-of-the-art DL algorithms forbearing fault applications. Specifically, the superiority of DL based methods over conventional ML methods are analyzed in terms of fault feature extraction and classification performances; many new functionalities enabled by DL techniques are also summarized. In addition, to obtain a more intuitive insight, a comparative study was conducted on the classification accuracy of different algorithms utilizing the open source Case Western Reserve University (CWRU) bearing dataset. Finally, to facilitate the transition on applying various DL algorithms to bearing fault diagnostics, detailed recommendations and suggestions are provided for specific application conditions such as the setup environment, the data size, and the number of sensors and sensor types.

(h) Swarm Intelligence: Swarm intelligence (SI) is an artificial intelligence technique based on the study of cooperation behaviors of simple individuals. The inspiration often comes from nature, especially biological systems (e.g., include ant colonies, bird flocking, animal herding, bacterial growth, fish schooling, bees gathering honey, etc.) in various decentralized systems. The population, which consists of simple individuals, can usually solve complex tasks in nature by individuals interacting locally with one another and with their environment. Although a simple individual's behavior is primarily characterized by autonomy, distributed functioning, and self-organizing capacities, local interactions among the individuals often lead to a global optimal. Therefore, swarm intelligence is a promising way to develop powerful solution methods for complex optimization, failure diagnosis and prognosis of complex systems in engineering \& technology management disciplines. Indeed, SI has been successfully applied to a variety of fields in fundamental research, engineering, industries, and social sciences. Recently, SI algorithms have attracted much attention of researchers and have also been applied successfully to solving optimization problems in mechanical engineering. However, for large and complex problems, SI algorithms often consume too much computation time due to the stochastic features of their searching approaches. Thus, there is a potential requirement to develop efficient algorithms that are able to find solutions under limited resources, time and money in real-world applications. The following topics are also included: 1) SI algorithms for scheduling of machinery production line, 2) Mechanical parameters adjustment based on SI, 3) Application of SI algorithms in mechanical fault diagnosis, 4) Application of SI algorithms in intelligent maintenance of machinery, 5) SI algorithms for study of transportation engineering, 6) Swarm intelligence and mechatronics, 7) Mechanical design. Some Examples of Swarm Intelligence algorithms are: 1) Particle swarm optimization (PSO), 2) Ant colony optimization, 3) Artificial bee colony algorithm, 4) Differential evolution, 5) The bees algorithm, 6) Artificial immune systems, 7) Bat algorithm, 8) Glowworm swarm optimization, 9) Gravitational search algorithm, 10) River Formation Dynamics, 11) Selfpropelled particles, 12) Stochastic diffusion search 13) Multi-swarm optimization. Hongxia Pan, Xiuye Wei \& Jinying Huang [35] applied the modified version of the PSO algorithm, a neural network system for gearbox fault diagnosis. After being trained by PSO algorithm, the neural network system was applied to fault diagnosis. Comparison of the diagnostic results between the PSO-based algorithm and the BP-based algorithm indicates that the network system based on PSO algorithm is of better training performance, and of less overall output error compared with those of the BP algorithm. It has been proved that the neural network system for fault diagnosis based on PSO algorithm is of higher probability of identifying multi-fault symptoms, and of rapid convergence and higher diagnosis accuracy, and that the neural network system for fault diagnosis expects a wide application in the field of mechanic fault diagnosis because of its higher searching efficiency. Qian-Jin Guoa, Hai-bin Yu \& Aidong $\mathrm{Xu}$ [71] present an intelligent methodology for diagnosing incipient faults in rotating machinery. In this fault diagnosis system, wavelet neural network techniques are used in combination with a new evolutionary learning algorithm. This new evolutionary learning algorithm is based on a hybrid of the constriction factor approach for particle swarm optimization (PSO) technique and the gradient descent (GD) technique, and is thus called HGDPSO. The HGDPSO is developed in such a way that a constriction factor approach for particle swarm optimization (CFA for PSO) is applied as a based level search, which can give a good direction to the optimal global region, and a local search gradient descent (GD) algorithm is used as a fine tuning to determine the 
optimal solution at the final. The effectiveness of the HGDPSO based WNN is demonstrated through the classification of the fault signals in rotating machinery. The simulated results show its feasibility and validity. P. S. Shelokar et al [85] propose PSACO (Particle Swarm Ant Colony Optimization) algorithm for highly nonconvex optimization problems. Both Particle Swarm Optimization (PSO) and Ant Colony Optimization (ACO) are co-operative, population-based global search swarm intelligence metaheuristics. PSO is inspired by social behavior of bird flocking or fish schooling, while ACO imitates foraging behavior of real life ants. In this study, the authors explore a simple pheromone-guided mechanism to improve the performance of PSO method for optimization of multimodal continuous functions. The proposed PSACO algorithm is tested on several benchmark functions from the usual literature. Numerical results comparisons with different metaheuristics demonstrate the effectiveness and efficiency of the proposed PSACO method. M. Luo, D. H. Zhang, L. L. Aw and F. L. Lewis [52] successfully identified precursory alarm sequence patterns for predicting equipment failures using ant colony-based algorithm. In industry, enormous files of historical data are collected from equipment monitoring prior to failures. The search for reliable precursory alarm patterns, that is, specific sequences of alarm events, in such data sets is a challenging task. The authors describes an algorithm for identifying precursory alarm patterns from historical measured event data containing numerous fault alarms and equipment states. The algorithm is based on modifications to ant colony optimization (ACO), which is an effective probabilistic learning method for finding shortest paths in large complex graphs. An actual industry application is used to verify the algorithm. Cloud computing means storing and accessing data and programs over the Internet instead of your computer's hard drive. The cloud is just a metaphor for the Internet. It goes back to the days of flowcharts and presentations that would represent the gigantic server-farm infrastructure of the Internet as nothing but a puffy, white cumulonimbus cloud, accepting connections and doling out information as it floats. For it to be considered "cloud computing," one need to access the data or the programs over the Internet, or at the very least, have that data synchronized with other information over the Web. Cloud computing can be done anywhere, anytime. A Swarm Intelligence based model for mobile cloud computing has been developed by Ahmed S. Salama [2]. O. Zedadra, et al [67] provide an initial understanding of the technical aspects of swarm intelligence algorithms and their potential use in IoTbased applications. They present the existing swarm intelligence-based algorithms with their main applications, then they present existing IoT-based systems that use SI-based algorithms. Finally, they discuss trends to bring together swarm intelligence and IoT-based systems. This review will pave the path for future studies to easily choose the appropriate SI-based algorithm for IoT-based systems. Lucija Brezočnik [51] et al performed a comprehensive literature review of SI algorithms and provide a detailed overview of 64 different SI algorithms for feature selection (FS), organized into eight major taxonomic categories. They propose a unified swarm intelligence (SI) framework and use it to explain different approaches to FS. Different methods, techniques, and their settings are explained, which have been used for various FS aspects. The datasets used most frequently for the evaluation of SI algorithms for FS are presented, as well as the most common application areas. The guidelines on how to develop SI approaches for FS are provided to support researchers and analysts in their data mining tasks and endeavors while existing issues and open questions are being discussed. In this manner, using the proposed framework and the provided explanations, one should be able to design an SI approach to be used for a specific FS problem. Guilherme Guerreiro et al [33] focus on a logistic process of car manufacturing, where batteries are unloaded from trucks to warehouse, and then to the point of fit, where they are assembled into the car. It presents a complete data-driven architecture, using a swarm approach for distributed data processing among all data stages, where processing nodes with different tasks and technologies can work cooperatively to complete a job.

(i) Distributed Intelligence: Distributed Intelligence (DI) is also known as Distributed Logic (DL). It refers to interconnected systems of entities working together to reason, plan, solve problems, think abstractly, comprehend ideas, language, learn and adapt to new situations. It covers intelligent processes, sub-systems, agents, humans, robots, smart sensors, signal processing systems, broadband and wireless systems. Distributed intelligence is widely used in many modern engineering/manufacturing industries and also in military applications. With the advent of Industry 4.0 (Fourth Industrial Revolution) the users would be able to do much more than understand their asset structure and relationships. Distributed intelligence will, in essence, be able to talk to each other and communicate knowledge-based information, users will be able to investigate and understand root cause failure mechanisms and performance efficiency of their assets in real-time in much greater depth than ever before. A number of tools, techniques and strategies are being developed and successfully employed in the interdisciplinary field of distributed intelligence. These are as follows: ECStar, a distributed rule-based learning system, Distributed Artificial Intelligence (DAI), Internet of Things (IoT), XpertRule is the intelligent brain of IoT, Minimum Available 
Distribution Assets, Swarm Intelligence, Semantic Web Services, Big Data and Cloud Computing, Global System for Mobile Communication (GSM), Bluetooth, Internet Protocol (IP), Reconfigurable Optical AddDrop Multiplexer (ROADM), Optical Spectrum Analyzer (OSA), Synchronous Ethernet (SyncE), IEEE 1588v2 Precision Time Protocol (PTP). Fred M. Discenzo Kenneth et al [19] provide specific examples of elements in the areas of Framework, Distributed Intelligent Modules, and Infrastructure for system-level integration. They emphasize a shift toward a new Maintenance Management Paradigm which has implications in many areas of the business including manufacturing scheduling, control, finance, inventory, quality, and asset management. According to the authors, implementation of the new Maintenance Management Paradigm will require three fundamental building blocks. First, is a framework that enables the efficient re-use of best-in-class diagnostic and prognostic software, hardware, and sensor modules. An open-system architecture will be fundamental to meeting this objective. Second, is the ability to rapidly deploy needed hardware and software elements in a reliable and cost-effective manner across distributed system components. Wireless, intelligent sensor nodes will play an important role in the deployment of future systems. And third, is the infrastructure and that will permit system level integration of an ensemble of distributed intelligent system elements to develop actionable diagnostic and prognostic information. Higher-level diagnostic and prognostic information will drive critical decision making to insure maximum system reliability, lowest operating cost, maximum revenue generation or mission success for example. Campos et al [14] reports the development of an emonitoring and maintenance system based on web technology and mobile device, PDA. Rarity of experts led to the application of artificial intelligence and later, distributed artificial intelligence for condition monitoring and diagnosis of machine condition. Recently, web technology along with wireless communication is emerging as a potent tool in maintenance. It facilitates obtaining of the desired information by the relevant personnel at any time wherever he may be. The paper first describes the web and mobile architecture that formed the basis of the system and ICT tools used to communicate among the different layers in the architecture/system and its various client machines. It is followed by the demonstration of the use of the system with a faulty bearing simulated signal. A mobile emulator was used to perfect the system for different requirements and the same was then tested on the PDA. Eduardo Gilabert \& Alexandre Voisin [24] presents a semantic web services platform ready to integrate intelligent processing capabilities according to open systems architecture for condition based maintenance (OSA-CBM) architecture.
This platform is part of a flexible communication infrastructure, nicknamed DynaWeb, where a generic wireless device is also being developed between novel sensors, smart PDAs and existing maintenance systems of companies. In a chapter on Distributed Intelligence published in a book on E-Maintenance, Asish Ghosh [3] starts with the description of an on-board chilled water supply system on a navy ship and how the robustness of the system be increased by distributing its control structure. Then, there is discussion on the need for decentralized structure, as the systems get increasingly complex. That is followed by a narrative on how computer software has evolved from simple programs with subroutines, to objects, and intelligent agents. Then, the advantages of intelligent agents and their applications are discussed in detail. Finally, the agentbased modelling and simulation techniques are described with an example. Paolo Gunet et al [68] describe the development of a simulation architecture for Gas-Turbine Engine Control and Health Monitoring. This architecture allows integration of advanced software technologies with conventional modelling techniques. The architecture is then used in the implementation of a complete Health Monitoring system that utilizes Case-Based Reasoning to achieve Fault Isolation and Intelligent Agents to achieve Fault Mitigation. Marek B. Zaremba \& Gérard Morel [62] aims to present a unified framework for integrating the methods and techniques related to intelligent systems in the context of design and control of modern manufacturing systems. Particular emphasis is placed on the methodologies relevant to distributed processing over the Internet. Following presentation of a spectrum of intelligent techniques, a framework for integrated analysis of these techniques at different levels in the context of intelligent manufacturing systems is discussed. Integration of methods of artificial intelligence is investigated primarily along two dimensions: the manufacturing product life-cycle dimension, and the organizational complexity dimension. It is shown that at different stages of the product life-cycle, different intelligent and knowledgeoriented techniques are used, mainly because of the varied levels of complexity associated with those stages. Distribution of the system architecture or system control is the most important factor in terms of demanding the use of the most up-to-date distributed intelligence technologies. A tool set for web-enabled design of distributed intelligent systems is presented. Finally, the issue of intelligence control is addressed. It is argued that the dominant criterion according to which the level of intelligence is selected in technological tasks is the required precision of the resulting operation, related to the degree of generalization required by the particular task. The control of knowledge in higher-level tasks has to be executed with a strong involvement of the human 
component in the feedback loop. In order to facilitate the human intervention, there is a need for readily available, user-transparent computing and telecommunications infrastructure. In its final part, the paper discusses currently emerging ubiquitous systems, which combine this type of infrastructure with new intelligent control systems based on a multi-sensory perception of the state of the controlled process and its environment to give us tools to manage information in a way that would be most natural and easy for the human operator. The growing instability of the business arena, advancements of the information and communication technologies, and increased competition determined manufacturing enterprises to change their way of pursuing business. As a consequence, new paradigms for manufacturing engineering systems have emerged. Claudia-Melania Chituc \& Francisco José Restivo [13] have presented preliminary results of an inter-disciplinary (on-going) research and development project focusing on the design, specification, performance modelling and implementation of an intelligent self-healing selfadaptable self-improving manufacturing engineering system (named wise manufacturing system). Main manufacturing paradigms are briefly presented, emphasizing their main strengths and weaknesses. The proposed system architecture towards a wise manufacturing engineering system is introduced, underlying main challenges and open issues. A cost model is also presented. Agent technology has been considered as an important approach for developing distributed intelligent manufacturing systems. A number of researchers have attempted to apply agent technology to manufacturing enterprise integration, supply chain management, manufacturing planning, scheduling and control, materials handling, and holonic manufacturing systems. Edge computing is a distributed computing paradigm which brings computation and data storage closer to the location where it is needed, to improve response times and save bandwidth. Monitoring the status of the facilities and detecting any faults are considered an important technology in a smart factory. Although the faults of machine can be analyzed in real time using collected data, it requires a large amount of computing resources to handle the massive data. A cloud server can be used to analyze the collected data, but it is more efficient to adopt the edge computing concept that employs edge devices located close to the facilities. Edge devices can improve data processing and analysis speed and reduce network costs. Donghyun Park et al [21] have constructed an edge device capable of collecting, processing, storing and analyzing data by using a single-board computer and a sensor. And, a fault detection model for machine is developed based on the long short-term memory (LSTM) recurrent neural networks. The proposed system called LiReD was implemented for an industrial robot manipulator and the LSTM-based fault detection model showed the best performance among six fault detection models. Small and medium sized manufacturers, as well as large original equipment manufacturers (OEMs), have faced an increasing need for the development of intelligent manufacturing machines with affordable sensing technologies and data-driven intelligence. Existing monitoring systems and prognostics approaches are not capable of collecting the large volumes of real-time data or building large-scale predictive models that are essential to achieving significant advances in cyber manufacturing. DazhongWu et al [22] introduce a new computational framework that enables remote real-time sensing, monitoring, and scalable high performance computing for diagnosis and prognosis. This framework utilizes wireless sensor networks, cloud computing, and machine learning. A proof-of-concept prototype is developed to demonstrate how the framework can enable manufacturers to monitor machine health conditions and generate predictive analytics. Experimental results are provided to demonstrate capabilities and utility of the framework such as how vibrations and energy consumption of pumps in a power plant and CNC machines in a factory floor can be monitored using a wireless sensor network. In addition, a machine learning algorithm, implemented on a public cloud, is used to predict tool wear in milling operations. Many cloud-based applications employ a data center as a central server to process data that is generated by edge devices, such as smartphones, tablets and wearables. This model places ever increasing demands on communication and computational infrastructure with inevitable adverse effect on Quality-of-Service and Experience. The concept of Edge Computing is predicated on moving some of this computational load towards the edge of the network to harness computational capabilities that are currently untapped in edge nodes, such as base stations, routers and switches. Blesson Varghes et al [11] considers the challenges and opportunities that arise out of this new direction in the computing landscape.

(j) Digital Twin Systems (DTS): It is widely acknowledged in both industry and academic publications that Dr. Michael Grieves, Chief Scientist of Advanced Manufacturing at the Florida Institute of Technology, originated the Digital Twin concept. The concept and model of the Digital Twin was publicly introduced in 2002 by Dr. Michael Grieves, then of the University of Michigan, at a Society of Manufacturing Engineers conference in Troy, Michigan. Dr. Grieves proposed the Digital Twin as the conceptual model underlying Product Lifecycle Management. The Digital Twin is a set of virtual information constructs that fully describes a potential or actual physical manufactured product from the micro atomic level to the macro geometrical level. At its optimum, any information that 
could be obtained from inspecting a physical manufactured product can be obtained from its Digital Twin. Digital twins integrate internet of things, artificial intelligence, machine learning and software analytics with spatial network graphs to create living digital simulation models that update and change as their physical counterparts change. A digital twin continuously learns and updates itself from multiple sources to represent its near real-time status, working condition or position. This learning system, learns from itself, using sensor data that conveys various aspects of its operating condition; from human experts, such as engineers with deep and relevant industry domain knowledge; from other similar machines; from other similar fleets of machines; and from the larger systems and environment of which it may be a part. A digital twin also integrates historical data from past machine usage to factor into its digital model. This pairing of the virtual and physical worlds allows analysis of data and monitoring of systems to head off problems before they even occur, prevent downtime, develop new opportunities and even plan for the future by using simulations. Prognostics and health management (PHM) is crucial in the lifecycle monitoring of a product, especially for complex equipment working in a harsh environment. Physics-based digital twins provide prognostics and health management which enables system optimization and predictive maintenance. The data can be used to optimize future designs, predict maintenance cycles, prevent spills, reduce downtime and improve throughput. A drone could monitor a pipeline and transmit its conditions to a digital twin that can predict maintenance cycles or improve future designs. In order to improve the accuracy and efficiency of PHM, digital twin (DT), an emerging technology to achieve physical-virtual convergence, is proposed by Fei Tao et al [28] for complex equipment. A general DT for complex equipment is first constructed, then a new method using DT driven PHM is proposed, making effective use of the interaction mechanism and fused data of DT. A case study of a wind turbine is used to illustrate the effectiveness of the proposed method. Modern aerospace industry is migrating from reactive to proactive and predictive maintenance to increase platform operational availability and efficiency, extend its useful life cycle and reduce its life cycle cost. Multiphysics modelling together with data-driven analytics generate a new paradigm called "Digital Twin." The digital twin is actually a living model of the physical asset or system, which continually adapts to operational changes based on the collected online data and information, and can forecast the future of the corresponding physical counterpart. Zheng Liu et al [105] review the overall framework to develop a digital twin coupled with the industrial Internet of Things technology to advance aerospace platforms autonomy. Data fusion techniques particularly play a significant role in the digital twin framework. The flow of information from raw data to high-level decision making is propelled by sensor-tosensor, sensor-to-model, and model-to-model fusion. This paper further discusses and identifies the role of data fusion in the digital twin framework for aircraft predictive maintenance. Wang Xiaodong et al [96] reviewed the various definitions and characteristics of digital twin and summarized the perspectives and process when using digital twins in prognostics and health management (PHM) areas. A digital twin-driven PHM framework of high-speed railway (Electrical Multiple Unit) maintenance has been proposed. ElisaNegri et al [23] aims at analyzing the definitions of the digital twin (DT) concept in scientific literature, retracing it from the initial conceptualization in the aerospace field, to the most recent interpretations in the manufacturing domain and more specifically in Industry 4.0 and smart manufacturing research. DT provides virtual representations of systems along their lifecycle. Optimizations and decisions making would then rely on the same data that are updated in real-time with the physical system, through synchronization enabled by sensors. The paper also proposes the definition of DT for Industry 4.0 manufacturing, elaborated by the European $\mathrm{H} 2020$ project MAYA, as a contribution to the research discussion about DT concept. Qinglin Qi et al [70] specifies and highlights how manufacturing services and digital twin are converged together and the various components of digital twin are used by manufacturers in the form of services. Jinjiang Wang et al [47] presents a Digital Twin reference model for rotating machinery fault diagnosis. The requirements for constructing the Digital Twin model are discussed, and a model updating scheme based on parameter sensitivity analysis is proposed to enhance the model adaptability. Experimental data are collected from a rotor system that emulates an unbalance fault and its progression. The data are then input to a Digital Twin model of the rotor system to investigate its ability of unbalance quantification and localization for fault diagnosis. The results show that the constructed Digital Twin rotor model enables accurate diagnosis and adaptive degradation analysis. Octavian Niculita, Obinna Nwora et al [66] presents a prognostic and health management (PHM) development process applied on a fuel oil system powering diesel engines typically used in various cruise and fishing vessels, dredgers, pipe laying vessels and large oil tankers. This process will hopefully enable future PHM solutions for maritime assets to be designed in a more formal and systematic way. Fei Tao et al [27] highlight the differences and correlation between cyber-physical systems (CPS) and digital twin (DTs) from multiple perspectives. Integrated Vehicle Health Management (IVHM) aims to support Condition-Based Maintenance (CBM) by 
monitoring, diagnosing, and prognosing the health of the host system. One of the technologies required by IVHM to carry out its objectives is the means to emulate the functioning of the host system, and the concept of a Digital Twin (DT) was introduced in aerospace IVHM to represent the functioning of such a complex system. Cordelia Mattuvarkuzhali Ezhilarasu et al [12] aims to discuss the role played by DT in the field of IVHM. A DT is the virtual representation of any physical product, that is used to project the functioning of the product at a given instance. The DT is used across the lifecycle of any product, and its output can be customized depending upon the area of application. The DT is currently popular in industry because of the technologies like sensors, cloud computing, Internet of Things, machine learning, and advanced software, which enabled its development. The authors discusses what encompasses a DT, the technologies that support the DT, its applications across industries, and its development in academia. They also highlight how a DT can combine with IVHM technology to assess the health of complex systems like an aircraft. Lastly, various challenges faced by industry during the implementation of a DT and some of the possible opportunities for future growth are also presented.

(k) Role of Biomimicry in machinery failure diagnosis and prognosis: Biomimetics is the study of nature and natural phenomena to understand the principles of underlying mechanisms, to obtain ideas from nature, and to apply concepts that may benefit science, engineering, and medicine. Examples of biomimetic studies include fluid-drag reduction swimsuits inspired by the structure of shark's skin, velcro fasteners modelled on burrs, shape of airplanes developed from the look of birds, and stable building structures copied from the backbone of turban shells. Diego F. Garcia et al [20] presents the design, development, and implementation of a bioinspired fault diagnosis scheme applied to a cold gas-based spacecraft prototype. The proposed framework relies on the artificial immune system metaphor with the goal of monitoring the health of the spacecraft by detecting subsystem upset conditions such as actuator malfunctions. A nonlinear dynamic inversion baseline controller with adaptation capabilities has been developed and implemented for attitude control of the spacecraft. The performance of the proposed health-monitoring scheme is determined by analyzing the system response under different actuator failures while the spacecraft is operated with and without adaptive augmentation. In both cases, the results show acceptable performance in terms of high detection activity and low false alarms with real-time capabilities. The application of this artificial intelligence-based scheme to aerospace systems will provide a high impact on space exploration by increasing mission protection and performance. SHI
Ding-ding and PAN Hong-Xia [82] introduces the principles and the algorithm model of the ant colony algorithm. Then the fault diagnosis system of neural network(NN)is established. ACA algorithm is used to train a NN for fault diagnosis of motor. The diagnostic results based on ACA are compared with ones of BP algorithm. contrast results of Network training show that fault diagnosis system based on ACA algorithm has a good identification probability of faults for multifault symptoms. ACA algorithm has faster convergence rate, higher accuracy and higher searching efficiency. Shrawasti Ganesh Sahare [86] investigated shark's skin ability to control the dynamic fluid environment through a novel inverse design by employing an industrial globe control valve consisting multi-stage continuous trim. Binitha S \& S Siva Sathya (2012) resents a broad overview of biologically inspired optimization algorithms, grouped by the biological field that inspired each and the areas where these algorithms have been most successfully. Paulo Leitãoa et al [69] provide an overview of some of the principles found in nature and biology and analyses the effectiveness of bio-inspired methods, which are used to enhance multi-agent systems to solve complex engineering problems, especially in the manufacturing field. An industrial automation case study is used to illustrate a bio-inspired method based on potential fields to dynamically route pallets. Exploring the symbiotic nature of biological systems can result in valuable knowledge for computer networks. Biologically inspired approaches to security in networks are interesting to evaluate because of the analogies between network security and survival of human body under pathogenic attacks. Wireless Sensor Network (WSN) is a network based on multiple lowcost, low-energy sensor nodes connected to physical signals. The network is made up of sensor nodes and gateways, where the server nodes acquire physical world data, while the gateway forwards the data to the end-user. While the spread of viruses in wired systems has been studied in-depth, applying trust in wireless sensor network nodes is an emerging area. Heena Rathore and Sushmita Jha [34] uses machine learning techniques to first differentiate between fraudulent and good nodes in the system. Next, it derives inspiration from the human immune system to present an idea of virtual antibodies in the system, to disable the fraudulent nodes in the system. Venkatesh Mahadevan and Frank Chiang [93] propose an energy efficient routing algorithm inspired from nature colonial scheme, its implementation and validation are also described in this paper. Details of the algorithm and its testing procedures are included. The proposed model is validated through simulations, demonstrating the network performance measurements such as delay, throughput and packet loss have been improved as a promising outcome. After decades of deploying cyber 
security systems, it is a well-known fact that the existing cyber infrastructure has numerous inherent limitations that make the maintenance of the current network security devices un-scalable and provide the adversary with asymmetric advantages. These limitations include: (1) difficulty in obtaining the global knowledge due to the lack of mutual interactions among network devices, (2) no sense of self-awareness, (3) absence of self-correcting/organizing mechanisms; for instance, error-prone and time-consuming manual configuration, which is not effective in real-time attack mitigation, (4) disability to diagnose mis-configuration and conflict resolution due to multiparty management of security infrastructure. Biological systems, on the other hand, have intrinsic appealing characteristics as a result of billions of years of evolution, such as adaptivity to varying environmental conditions, inherent resiliency to failures and damages, successful and collaborative operation on the basis of a limited set of rules with global intelligence (which is larger than superposition of individuals). Usman Rauf [92] has reviewed the existing bio-inspired approaches that have been used toward addressing the aforementioned issues and evaluate them accordingly. We also aim to provide information about the intrinsic potential of existing bioinspired techniques which has not been explored yet, for improving cyber security.

(1) Malicious application of Artificial Intelligence: Artificial intelligence and machine learning capabilities are growing at an unprecedented rate. These technologies have many widely beneficial applications in the multi-discipline of condition monitoring and diagnostic engineering management and countless applications ranging from machine translation to medical image analysis. Less attention has historically been paid to the ways in which artificial intelligence can be used maliciously. There are many studies (Rory Cellan-Jones [72], Vincent C. Müller [64], Taylor Meek et al [89], Miles Brundage et al [9], Peter J. Bentley et al [8], Bostrom, Nick, and Eliezer Yudkowsky [4]) reporting the landscape of potential security threats from malicious uses of artificial intelligence technologies, and ways to better forecast, prevent, and mitigate these threats. Malicious use of AI could threaten digital security (e.g. through criminals training machines to hack or socially engineer victims at human or superhuman levels of performance), physical security (e.g. non-state actors weaponizing consumer drones), and political security (e.g. through privacy-eliminating surveillance, profiling, and repression, or through automated and targeted disinformation campaigns).

\section{Conclusion}

Computer science defines AI research as the study of "intelligent agents" or any device that perceives its environment and takes actions that maximize its chance of successfully achieving its goals. A more elaborate definition characterizes AI as “a system's ability to correctly interpret external data, to learn from such data, and to use those learnings to achieve specific goals and tasks through flexible adaptation." Artificial Intelligence will change the way how humans communicate with machines, and between each other. More and more artificially and virtually created worlds extend our capabilities, and AI transforms user-experience towards more smart systems. AI research has developed methods for dealing with uncertain or incomplete information, employing concepts from different disciplines. Knowledge representation and knowledge engineering are central to classical AI research. AI has developed many tools (such as Search and optimization, logic, Probabilistic methods for uncertain reasoning, Classifiers and statistical learning methods, Artificial neural networks, Deep feedforward neural networks, Deep recurrent neural networks, Genetic Algorithms, Case Based Reasoning, Machine Learning, Digital Twin Systems, etc.) to solve the most difficult problems encountered in many areas such as, Condition Monitoring and Diagnostic Engineering Management, Prognostics and Health Management, Healthcare, Automotive, Finance and economics, Cybersecurity, Government, Law-related professions, defense, hospitality, audit, advertising, art, philosophy and ethics. Max Tegmark, President of the Future of Life Institute said "Everything we love about civilization is a product of intelligence, so amplifying our human intelligence with artificial intelligence has the potential of helping civilization flourish like never before as long as we manage to keep the technology beneficial." However, Stephen Hawking, Elon Musk, Steve Wozniak, Bill Gates, and many other big names in science and technology have recently expressed concern in the media and via open letters about the risks posed by AI, joined by many leading AI researchers. The smartest artificial intelligent machine may be able to diagnose complex problems and recommend actions to overcome many insurmountable problems in any organization. Artificial Intelligence is more oriented to several form of collective intelligence. Like everything else, collective intelligence has its own merits and demerits.

\section{References}

[1] Arslan, T. S., Bottaci, L. and Taylor, G. E. (1993), "A fault dictionary based expert system for failure diagnosis in a multiple-PCB environment", Engineering Applications of Artificial Intelligence, 6(5), pp. 447-456.

[2] Ahmed S. Salama (2015). A Swarm Intelligence Based Model for Mobile Cloud Computing. I. J. Information Technology and Computer Science, 02, 28 -34. http://www.mecspress.org/ijitcs/ijitcs-v7-n2/IJITCS-V7-N2-4.pdf

[3] Asish Ghosh (2015). Dynamic Systems for Everyone. Published by Springer. http://link.springer.com/chapter/10.1007/978-3-319-107356_7?no-access $=$ true 
[4] Bostrom, Nick, and Eliezer Yudkowsky. "The Ethics of Artificial Intelligence." In Cambridge Handbook of Artificial Intelligence, edited by Keith Frankish and William Ramsey. New York: Cambridge University Press.

[5] Branicky, M. S. (1995) Studies in Hybrid Systems: Modeling, Analysis, and Control. ScD thesis, Massachusetts Institute of Technology, Cambridge, MA

[6] N Belu, L M Ionescu and N Rachieru. (2019). Risk-cost model for FMEA approach through Genetic algorithms -A case study in automotive industry. IOP Conf. Series: Materials Science and Engineering564 (2019) 012102.

[7] Batanov, D. B., Nagarur, N. and Nitikhunkasem, P. (1993), "EXPERT-MM: A knowledge-based system for maintenance management", Artificial Intelligence in Engineering, Vol. 8, pp. 283-291.

[8] Peter J. Bentley et al (2018). Should we fear artificial intelligence? Published by European Parlimentary Research Service (EPRS), Scientific Forecast Unit (STOA) in March 2018.

[9] Miles Brundage et al (2018). The Malicious Use of Artificial Intelligence: Forecasting, Prevention, and Mitigation. https://arxiv.org/ftp/arxiv/papers/1802/1802.07228.pdf

[10] Binitha S \& S Siva Sathya (2012). A Survey of Bio inspired Optimization Algorithms. International Journal of Soft Computing and Engineering (IJSCE) ISSN: 2231-2307, Volume-2, Issue-2.

[11] Blesson Varghese, Nan Wang, Sakil Barbhuiya, Peter Kilpatrick and Dimitrios S. Nikolopoulos (2016). Challenges and Opportunities in Edge Computing. https://arxiv.org/pdf/1609.01967.pdf

[12] Cordelia Mattuvarkuzhali Ezhilarasu, Zakwan Skaf and Ian K Jennions (2019). Understanding the role of a Digital Twin in Integrated Vehicle Health Management (IVHM). Published in: 2019 IEEE International Conference on Systems, Man and Cybernetics (SMC) held in Bari, Italy held during 6-9 Oct. 2019.

[13] Claudia-Melania Chituc and Francisco José Restivo (2009). Challenges and Trends in Distributed Manufacturing Systems: Are wise engineering systems the ultimate answer? Second International Symposium on Engineering Systems MIT, Cambridge, Massachusetts, June 15-17.

[14] Campos Jaime; Jantunen, Erkki; Prakash Om (2007). Development of a Maintenance System Based on Web and Mobile Technologies. Journal of International Technology and Information Management (https://www.questia.com/read/1G1178945972/development-of-a-maintenance-system-based-onweb-and)

[15] Cordelia Mattuvarkuzhali Ezhilarasu, ZakwanSkaf and Ian K. Jennions. (2019). The application of reasoning to aerospace Integrated Vehicle Health Management (IVHM): Challenges and opportunities. Progress in Aerospace Sciences, Volume 105, February, Pages 60-73

[16] Chang, S. I. and Ho, E. S. (1999), "A two-stage neural network approach for process variance change detection and classification", International Journal of Production Research, 37 (7), pp. 1581-1599.

[17] Clark, G., Mehta, P. and Thomson, T. (1992), "Application of knowledge-based systems to optimised building management maintenance", Lecture notes in Artificial Intelligence, Vol. 604, pp. $69-78$.
[18] Dedeakayogullari, I. and Buma, K. N. (1999), "The determination of mean and/or variance shifts with artificial neural networks", International Journal of Production Research, 37 (10), pp. 2191-2200.

[19] M. Discenzo Kenneth, Kenneth A. Loparo, Dukki Chung and Allen Twarowski (2001). Intelligent Sensor Nodes Enable A New Generation of Machinery Diagnostics And Prognostics. Proceedings of the 55th Meeting of the Society for Machinery Failure Prevention Technology. Virginia Beach, Virginia, April 2 -5, 2001 (http://dtic.mil/dtic/tr/fulltext/u2/p013512.pdf)

[20] Diego F. Garcia, Andres E. Perez, Hever Moncayo, Karina Rivera, Yomary Betancur, Michael DuPuis and Robert P. Mueller (2018). Spacecraft Heath Monitoring Using a Biomimetic Fault Diagnosis Scheme. Published Online:12 Mar 2018https://doi.org/10.2514/1.I010612.

[21] Donghyun Park, Seulgi Kim, Yelin An, and Jae-Yoon Jung (2018). LiReD: A Light-Weight Real-Time Fault Detection System for Edge Computing Using LSTM Recurrent Neural Networks. Sensors (Basel). Jul; 18 (7): 2110.

[22] DazhongWu, Shaopeng Liu, Li Zhang, Janis Terpenny, Robert X. Gao, Thomas Kurfess, Judith A. Guzzo (2017). A fog computing-based framework for process monitoring and prognosis in cyber-manufacturing. Journal of Manufacturing Systems 43, 25-34.

[23] ElisaNegri, LucaFumagalli and MarcoMacchi (2017). A Review of the Roles of Digital Twin in CPS-based Production Systems. Procedia Manufacturing. Volume 11, 2017, Pages 939-948.

[24] Eduardo Gilabert \& Alexandre Voisin. (2010). Semantic Web Services for Distributed Intelligence. Chapter 11 from a book on E-Maintenance Edited by Kenneth Holmberg, Adam Adgar Aitor Arnaiz, Erkki Jantunen, Julien Mascolo, Samir Mekid, published by Springer. http://link.springer.com/chapter/10.1007/978-1-84996-205$6 \_11$.

[25] Edmundas Kazimieras Zavadskas, Edmundas Kazimieras Zavadskas, Jurgita Antucheviciene, Hojjat Adeli, Zenonas Turskis, Hojjat Adeli. Hybrid Multiple Criteria Decision Making Methods: A Review of Applications in Engineering. http://scientiairanica.sharif.edu/article_2093.html

[26] Erfan Ahadi, Mostafa Larky and Mohammad Riahi. (2018). Applications of Artificial Intelligence on Prognostics of Rotating Machineries. The 26th Annual International Conference of Iranian Society of Mechanical EngineersISME2018 24-26 April, 2018, School of Mechanical Engineering, Semnan University, Semnan, Iran.

[27] Fei Tao, Qinglin Qi, Lihui Wang and A. Y. C. Ne (2019). Digital Twins and Cyber-Physical Systems toward Smart Manufacturing and Industry 4.0: Correlation and Comparison. Engineering, Volume 5, Issue 4, August 2019, Pages 653-661.

[28] Fei Tao, Meng Zhang, Yushan Liu and Andrew Y C Nee (2018). Digital twin driven prognostics and health management for complex equipment. CIRP Annals, Volume 67, Issue 1, 2018, Pages 169-172.

[29] Frank, P. M. and Koppen-Seliger, B. (1997), "New developments of AI in fault diagnosis", Engineering Applications of Artificial Intelligence, 10 (1), pp. 3-14. 
[30] Fujikawa, S. and Ishii, K. (1995), "Diagnostic expert systems for defect in forged parts", Journal of Intelligent Manufacturing, 6(3), pp. 163-1733.

[31] Guh, R.S. and Tannock, J. D. T. (1999), "Recognition of control chart concurrent patterns using a neural network approach", International Journal of Production Research, 37(8), pp. 1745-1765.

[32] Gollu, A., and Varaiya, P. P. (1989) Hybrid dynamical systems In: Proc. IEEE Conf. Decision and Control, pp. 2708-2712. Tampa, FL.

[33] Guilherme Guerreiro, Ruben Costa, Paulo Figueiras, Diogo Graça and Ricardo Jardim-Gonçalves (2019). A Self-Adapted Swarm Architecture to Handle Big Data for "Factories of the Future". IFAC-Papers On Line, Volume 52, Issue 13, Pages 916-921.

[34] Heena Rathore and Sushmita Jha (2013). Bio-inspired machine learning based Wireless Sensor Network security. Published in: 2013 World Congress on Nature and Biologically Inspired Computing. DOI: 10.1109/NaBIC.2013.6617852.

[35] Hongxia Pan, Xiuye Wei \& Jinying Huang. Application of PSO Algorithm to Gearbox Fault Diagnosis.. School of Mechanical Engineering and Automation, North University of China, Taiyuan 030051, P. R. China.

[36] Hen Zhang, Shibo Zhang, Bingnan Wang, and Thomas G. Habetler. Machine Learning and Deep LearningAlgorithms for Bearing Fault Diagnostics- A Comprehensive Review. https://arxiv.org/pdf/1901.08247.pdf

[37] Hosameldin Ahmed and Asoke K Nandi (2019). Condition Monitoring with Vibration Signals; Compressive Sampling and Learning Algorithms for Rotating Machines. Wiley. ISBN 9781119544623 .

[38] Haitham Ramadan (2017). New approach to power transformer asset management and life assessment using fuzzy logic techniques. Published in: 2017 Nineteenth International Middle East Power Systems Conference (MEPCON) held in Cairo, Egypt. https://ieeexplore.ieee.org/document/8301287

[39] Hans R. DePold and F. Douglas Gass. (1998). The Application of Expert Systems and Neural Networks to Gas Turbine Prognostics and Diagnostics. Presented at the International Gas Turbine \& Aeroengine Congress \& Exhibition Stockholm, Sweden during June 2-June 5.

[40] Ho, E. S. and Chang, S. I. (1999), "An integrated neural network approach for simultaneous monitoring of process mean and variance shift - a comparative study", International Journal of Production Research, 37(8), pp. 1881-1901.

[41] Ion Matei, Anurag Ganguli, Tomonori Honda and Johan de Kleer. The Case for a Hybrid Approach to Diagnosis: A Railway Switch. Proceedings of the 26th International Workshop on Principles of Diagnosis. https://www.researchgate.net/profile/Tomonori_Honda/publicati on/305731351 The Case for a Hybrid Approach to Diagno

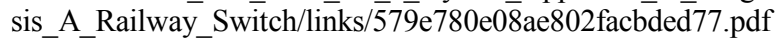

[42] Ivica Petrović, Lajos Jozsalajos and Zoran Baus. (2015). Use of Fuzzy Logic Systems for Assessment of Primary Faults. Journal of Electrical Engineering, Volume 66: Issue 5.

[43] Jianbin Xiong, Qinghua Zhang, Qiong Liang, Hongbin Zhu and Haiying L. (2018). Combining the Multi-Genetic Algorithm and Support Vector Machine for Fault Diagnosis of
Bearings. Shock and Vibration. Volume 2018 Article ID 3091618 | 13 pages.

[44] Jun-jie Chen, Jiang-li Duan, Bao-lu Gao and Ting-ting Lu (2010). The application of CBR and grey correlation in fault diagnosis system. Published in: 2010 International Conference on Computer Application and System Modeling (ICCASM 2010).

[45] Jan Lunze and Françoise Lamnabhi-Lagarrigue (2009) Handbook of Hybrid Systems Control: Theory, Tools, Applications,. Published by Cambridge University Press.

[46] J. Jeon (2000). The development of a hybrid intelligent maintenance optimization system (HIMOS).

[47] Jinjiang Wang, Lunkuan Ye, Robert X. Gao, Chen Li \& Laibin Zhang (2019). Digital Twin for rotating machinery fault diagnosis in smart manufacturing. International Journal of Production Research. Volume 57, 2019 - Issue 12: Special Issue: Sustainable Cybernetic Manufacturing.

[48] Khoo, L. P., Ang, C. L. and Zhang, J. (1999), “An IDEF0 model-based intelligent fault diagnosis system for manufacturing systems", International Journal of Production Research, 37 (1), pp. 35-48.

[49] Kim, T. and Kumara, S. R. T. (1997), "Boundary defect recognition using neural networks", International Journal of Production Research, 35(9), pp. 2397-2412.

[50] Kobbacy, K. A. H., Proudlove, N. C. and Harper, M. A. (1995), "Towards an intelligent maintenance optimisation system", Journal of the Operational Research Society, Vol.46, pp. 831853.

[51] Lucija Brezočnik, Iztok Fister, Jr. and Vili Podgorelec. (2018). Swarm Intelligence Algorithms for Feature Selection: A

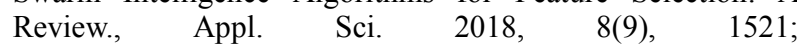
https://doi.org/10.3390/app8091521.

[52] M. Luo, D. H. Zhang, L. L. Aw and F. L. Lewis (2010), "Identification of Precursory Alarm Sequence Patterns for Predicting Equipment Failures Using Ant Colony-Based Algorithm ", International Journal of COMADEM, vol. 13(2), pp. 34-45 (APR-2010).

[53] Linxia Liao and Hyung-il Ahn. Combining Deep Learning and Survival Analysis for Asset Health Management. International Journal of Prognostics and Health Management, ISSN21532648, 2016020.

[54] W. Labib, G. B. Williams and R. F. O'Connor (1998) An Intelligent Maintenance Model (System): An Application of the Analytic Hierarchy Process and a Fuzzy Logic Rule-Based Controller. The Journal of the Operational Research Society. Vol. 49, No. 7, Intelligent Management Systems in Operations (Jul., 1998).

[55] Lei Lu, JihongYan and YueMeng. (2016). Dynamic Genetic Algorithm-based Feature Selection Scheme for Machine Health Prognostics. Procedia CIRP, Volume 56, Pages 316-320.

[56] María Matilde García Lorenzo and Rafael Estebán Bello Pérez. (1996). A model and its different applications to case-based reasoning. Publication: Knowledge-Based Systems November 1996 https://doi.org/10.1016/S0950-7051(96)01058-1

[57] Mehul Ved (2018). Feature Selection and Feature Extraction in Machine Learning: An Overview.https://medium.com/@mehulved1503/featureselection-and-feature-extraction-in-machine-learning-anoverview-57891c595e96 
[58] Matteo D. L. Dalla Vedova, Alfio Germanà, Pier Carlo Berri and Paolo Maggiore. (2019). Model-Based Fault Detection and Identification for Prognostics of Electromechanical Actuators Using Genetic Algorithms. Aerospace 2019, 6(9), 94.

[59] M Muharam and M Latif. (2019). Design of poka-yoke system based on fuzzy neural network for rotary-machinery monitoring. IOP Conf. Series: Materials Science and $\begin{array}{lll}\text { Engineering602 } & \text { (2019) }\end{array}$ Publishingdoi:10.1088/1757-899X/602/1/012003

[60] Maurizio Bevilacqua, Marcello Braglia, Marco Frosolini and Roberto Montanari (2005). Failure rate prediction with artificial neural networks, al of Quality in Maintenance Engineering 11(3):279-294 $\cdot$ September

[61] Mateusz Dybkowski1 and Kamil Klimkowski. (2019). Artificial Neural Network Application for Current Sensors Fault Detection in the Vector Controlled Induction Motor Drive. Sensors (Basel). 2019 Feb; 19(3): 571

[62] Marek B. Zaremba, Gérard Morel (2003). Integration and control of intelligence in distributed manufacturing. Journal of Intelligent Manufacturing, Volume 14, Issue 1, pp 25-42.

[63] Maryam M Najafabadi, Flavio Villanustre, Taghi M Khoshgoftaar, Naeem Seliya, Randall Wald \& Edin Muharemagic (2015). Deep learning applications and challenges in big data analytics. Journal of Big Data volume 2, Article number: 1 (2015).

[64] Vincent C. Müller (2015). 'Editorial: Risks of artificial intelligence', in Vincent C. Müller (ed.), Risks of general intelligence (London: CRC Press - Chapman \& Hall).

[65] Oluseun Omotola Aremu, Adrià Salvador Palau, Ajith Kumar Parlikad, David Hyland-Wood (2018). Structuring Data for Intelligent Predictive Maintenance in Asset Management, IFAC-Papers On Line, Volume 51, Issue 11, 2018, Pages 514519.

[66] Octavian Niculita, Obinna Nwora and Zakwan Skaf (2017). Towards Design of Prognostics and Health Management Solutions for Maritime Asset. Procedia CIRP. Volume 59, 2017, Pages 122-132.

[67] Ouarda Zedadra, Antonio Guerrieri, Nicolas Jouandeau and Giandomenico Spezzano. (2018). Swarm intelligence-based algorithms within IoT-based systems: A review, J. Parallel Distributed Computing. 122, August, https://doi.org/10.1016/j.jpdc.2018.08.007

[68] Paolo Gunet, Andrew Mills and Haydn Thompson. A Distributed Intelligent Agent Architecture for Gas-Turbine Engine Health Management. Published by American Institute of Aeronautics and Astronautics. http://web.eecs.umich.edu/ soar/sitemaker/docs/pubs/Distrib Intell_Agent_Arch_Gas-Turbine_Engine_Management.pdf

[69] Paulo Leitãoa, José Barbosa \& Damien Trentesaux (2012). Bio-inspired multi-agent systems for reconfigurable manufacturing systems. Engineering Applications of Artificial Intelligence, Volume 25, Issue 5, August, Pages 934-944.

[70] Qinglin Qi, FeiTao, Ying Zuo and Dongming Zhao (2018). Digital Twin Service towards Smart Manufacturing. Procedia CIRP, Volume 72, 2018, Pages 237-242.

[71] Qian-jin Guoa, Hai-bin Yu \& Ai-dong Xu (2006). A hybrid PSO-GD based intelligent method for machine diagnosis. Digital Signal Processing, Volume 16, Issue 4, July, Pages
$402-418$.

[72] Rory Cellan-Jones (2014), Stephen Hawking warns artificial intelligence could end mankind. https://www.bbc.co.uk/news/technology-30290540.

[73] Rafik Mahdaoui and Leila Hayet Mouss. (2012). A TSK-Type Recurrent Neuro-Fuzzy Systems for Fault Prognosis. Journal of Software Engineering and Applications, 2012, 5, 477-482.

[74] Ransing, R. S. and Lewis, R. W. (1997), "A semantically constrained neural network for manufacturing diagnosis", International Journal of Production Research, 35(9), pp.26392660 .

[75] Rene Peinl (2017). Knowledge Management 4.0 - Lessons Learned from IT Trends. Conference: Professional Knowledge Management (WM 2017), At Karlsruhe.

[76] Rowland, J. G. and Jain L. C. (1993), "Knowledge-based systems for instrumentation diagnosis, system configuration and circuit and system design", Engineering Applications of Artificial Intelligence, 6 (5), pp. 437-446.

[77] Timothy. J Ross (2009). Fuzzy Logic With Engineering Applications.

https://www.researchgate.net/publication/243658533_Fuzzy_ Logic_With_Engineering_Applications

[78] Shu-hsien Liao (2003). Knowledge management technologies and applications - literature review from 1995 to 2002. Expert Systems with Applications. Volume 25, Issue 2, August 2003, Pages 155-164.

[79] Su, C. T., Chang, C. A. and Tien, F. C. (1995), "Neural networks for precision measurement in computer vision systems", Computers in Industry, 27, pp. 225-236.

[80] A. Soliman, G. Rizzoni and Y. W. Kim (1999). Diagnosis of an automotive emission control system using fuzzy inference. Control Engineering Practice, Volume 7, Issue 2, February 1999, Pages 209-216.

[81] B. Samanta, K. R. Al-Balushi and S. A. Al-Araimi (2003). Artificial neural networks and support vector machines with genetic algorithm for bearing fault detection. Engineering Applications of Artificial Intelligence. Volume 16, Issues 7-8, October-December 2003, Pages 657-665.

[82] SHI Ding-ding and PAN Hong-xia (2009). Ant Colony Algorithm Application to the Fault Diagnosis of Motor. Large Electric Machine and Hydraulic Turbine. 2009-01

[83] Rui G. Silva, Steve J. Wilcox and Robert L. Reuben. (2001). Development of a system for monitoring tool wear using artificial intelligence techniques. Published in the proceedings of the ASME International Mechanical Engineering Congress and Exposition.

[84] Samir Khan and Takehisa Yairi (2018) A review on the application of deep learning in system health management. Mechanical Systems and Signal Processing, Volume 107, July 2018, Pages 241-265.

[85] P. S. Shelokar, Patrick Siarry, V. K. Jayaraman and B. D. Kulkarni. (2007). Particle swarm and ant colony algorithms hybridized for improved continuous optimization. Applied Mathematics and Computation, Volume 188, Issue 1, 1 May 2007, Pages 129-142. 
[86] Shrawasti Ganesh Sahare (2020). Inverse design of functional surfaces for prescribed simple and complex flow characteristics. PhD Thesis. University of Huddersfield. UK.

[87] Tien, F. C. and Chang, C. A. (1999), "Using neural networks for 3D measurement in stereo-vision inspection systems", International Journal of Production Research, 37(9), pp. 19351948.

[88] Toyosi T. Ademujimi, Michael P. Brundage and Vittaldas V. Prabhu. A review of current machine learning techniques used in manufacturing diagnosis. https://tsapps.nist.gov/publication/get_pdf.cfm?pub_id=92330 9.

[89] Taylor Meek et al (2016), Managing the Ethical and Risk Implications of Rapid Advances in Artificial Intelligence: A Literature Review. 2016 Proceedings of PICMET '16: Technology Management for Social Innovation. https://pdxscholar.library.pdx.edu/cgi/viewcontent.cgi?article= $1113 \&$ context $=$ etm_fac.

[90] Urko Leturiondo Zubizarreta. (2016). Hybrid modelling in condition monitoring. PhD Thesis. Lulea University of Technology, Sweden.

[91] Urko Leturiondo, OscarSalgado, Lorenzo Ciani, Diego Galar and Marcantonio Catelani. (2017). Architecture for hybrid modelling and its application to diagnosis and prognosis with missing data. Measurement, Volume 108, October 2017, Pages 152-162.

[92] Usman Rauf (2018). A Taxonomy of Bio-Inspired Cyber Security Approaches: Existing Techniques and Future Directions. Computer Engineering and Computer Science, February.

[93] Venkatesh Mahadevan and Frank Chiang (2010). iACO: A Bio-inspired Power Efficient Routing Scheme for Sensor Networks. International Journal of Computer Theory and Engineering, Vol. 2, No. 6, December, 1793-8201

[94] Wang, C. and Huang, S Z. (1997), "A refined flexible inspection method for identifying surface flaws using the skeleton and neural network", International Journal of Production Research, 35 (9), pp. 2493-2507.

[95] Wilson Q. Wang, M. Farid, Golnaraghi and FathyIsmail (2004). Prognosis of machine health condition using neuro- fuzzy systems. Mechanical Systems and Signal Processing. Volume 18, Issue 4, July 2004, Pages 813-831.

[96] Wang Xiaodong, Liu Feng, Ren Junhua and Liang Rongyu (2019). A Survey of Digital Twin Technology. Published by Springer Nature Singapore Pte Ltd in Recent Trends in Intelligent Computing, Communication and Devices. Edited by V. Jain, Srikanta Patnaik and Florin Popentiu Vladicescu.

[97] Xia, Q. J. and Rao, M. (1999), Dynamic case-based reasoning for process operation support systems, Engineering Applications of Artificial Intelligence, 12 (3), pp. 343-361.

[98] Yang Lu (2017). Industry 4.0: A survey on technologies, applications and open research issues. Journal of Industrial Information Integration Volume 6, June 2017, Pages 1-10.

[99] Zhang, F. and Jardine, A. K. S. (1997), “A smart maintenance decision system", Proceedings of the European Conference on Intelligent Management Systems Operations, pp. 79-86.

[100] Zhang, H. C. and Huang S. H (1995), “Application of neural networks in manufacturing: a state of the art", International Journal of Production Research, 33(3), pp. 705-728.

[101]Zhe Li, Yi Wang \& Ke-Sheng Wang. (2017). Intelligent predictive maintenance for fault diagnosis and prognosis in machine centers: Industry 4.0 scenario. Advances in Manufacturing volume 5, pages 377-387.

[102] Ziyan Wen, J. Cardillo, Jacob Crossman and Yi Murphey (2003). Case Base Reasoning in Vehicle Fault Diagnostics. Conference: Neural Networks, 2003. Proceedings of the International Joint Conference on, Volume: 4.

[103] Zhiwang Zhong, Tianhua Xu, Feng Wang, and Tao Tang (2018). Text Case-Based Reasoning Framework for Fault Diagnosis and Predication by Cloud Computing.

[104] Mathematical Problems in Engineering, Article ID 9464971, 10 pages $\mathrm{Zhe} \mathrm{Li}$, Jingyue $\mathrm{Li}$, Yi Wang and Kesheng Wang (2019). A deep learning approach for anomaly detection based on SAE and LSTM in mechanical equipment. The International Journal of Advanced Manufacturing Technology volume 103, pages499-510(2019).

[105] Zheng Liu, Norbert Meyendorf and Nezih Mrad (2018). The Role of Data Fusion in Predictive Maintenance Using Digital Twin. 44th Annual Review of Progress in Quantitative Nondestructive Evaluation, Volume 37. 